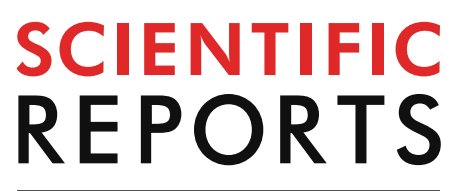

natureresearch

Check for updates

\title{
Environmentally friendly quantum-dot color filters for ultra-high-definition liquid crystal displays
}

\author{
Yun-Hyuk Ko ${ }^{1,2,3}$, Prem Prabhakaran ${ }^{1,3}$, Sinil Choi ${ }^{1}$, Gyeong-Ju Kim ${ }^{1}$, Changhee Lee ${ }^{2 \bowtie} \&$ \\ Kwang-Sup Lee ${ }^{1 \bowtie}$
}

This work reports the synthesis and application of highly tuned cadmium-free green and red InPZnSe ${ }_{1-x} S_{x} / Z n S$ quantum dots (ODs) in OD enhanced liquid crystal displays (LCD). The emissions of the quantum dots were synthetically tuned to sharp emissions at low full-width at half maximum. The ODs were incorporated in LCD devices as quantum dot enhancement film (ODEF) or as a quantum dot incorporated color filter (QDCF). Synthetic tuning of the gradient inter-shell in the QDs leads to reduced full width at half-maximum, resulting in sharp green and red emissions from both types of devices. The application of the same QDs to devices using these different integration techniques shows the superiority of QDCF devices over QDEF ones. The RGB color gamut of a ODCF-LCD was $81.4 \%$ of REC. 2020 in the CIE 1931 color space compared to $71.2 \%$ obtained for a ODEF-LCD display. The improved performance of ODCF was mainly due to the optimal interactions between the green $\mathrm{QDs}$ and the green color filter. The superior performance of cadmium-free $\operatorname{InPZnSe_{1-x}} \mathrm{S}_{\mathrm{x}} / \mathrm{ZnS} \mathrm{ODCFs}$ in LCDs make them well-suited for ultra-high-definition TV formats.

Semiconductor quantum dots (QDs) are important emissive materials for optoelectronic applications because of their bright and well-defined emission properties. The ubiquitous potential of these materials has been established by a variety of demonstrated applications, from bio-imaging to displays and solar cells ${ }^{1-3}$. Despite such demonstrations and millions of dollars spent on research there are only a handful of commercialized products containing quantum dots available in the market. The reason for this is the toxicity of the elements contained in the widely studied II-VI QDs of which cadmium is a main component. Cadmium (Cd)-containing QDs are easy to synthesize and exhibit excellent optical properties. However, use of Cd-based materials are restricted in commercial products by the restriction of hazardous substances directive (RoHS). The safer alternative to II-VI QDs are III-V QDs based on Indium. The nucleation growth of indium (In)-based quantum dots has proven to be a difficult task because of the different nature of bonding in these materials ${ }^{4}$. Last decade has seen rapid advances in the understanding of III-V quantum dots, leading to the eventual commercialization liquid crystal displays (LCD) with quantum dot enhancement films (QDEF) often referred to as QLED TVs.

Quantum dot enhanced displays have high color gamut and enables ultra-high definition TV (UHDTV) formats like $4 \mathrm{~K}, 8 \mathrm{~K}$ and HDR. At the end of 2018 , $4 \mathrm{~K}$ TV technology was still being adapted by broadcasters and streaming services around the world. Currently both $8 \mathrm{~K}$ and HDR formats suffer from lack of content due to the scarcity of terrestrial transmission of these formats. There are plans to highlight $8 \mathrm{~K}$ TV technology during the summer Olympics in Tokyo (Tokyo 2020, currently postponed due to covid-19 pandemic). Widespread viewing of $8 \mathrm{~K}$ content of this event would boost the spread of $8 \mathrm{~K}$ technology worldwide. With $4 \mathrm{~K}$ still going through experimental implementation in many countries, it is safe to say that we are a good part of a decade away from acceptance of $8 \mathrm{~K}$ as a widespread broadcast format. Both these UHDTV formats with their high bandwidths are poised to dictate the visual experience and aural detail of how we will be informed and entertained in the near

${ }^{1}$ Department of Advanced Materials, Hannam University, Yuseong-daro, Yuseong-gu, Daejeon 34054, Republic of Korea. 'Display Research Center, Samsung Display, Samsung-ro, Giheung-gu, Yongin-si, Gyeonggi-do 17113, Republic of Korea. ${ }^{3}$ These authors contributed equally: Yun-Hyuk Ko and Prem Prabhakaran. ${ }^{\square}$ email: chlee7@ samsung.com; kslee@hnu.kr 
(a) LED TV: White LED backlit LCD TV (CF)

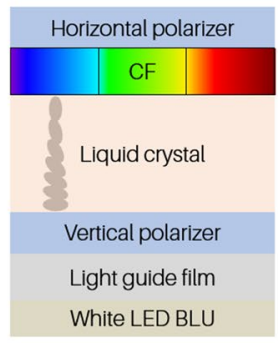

(b)

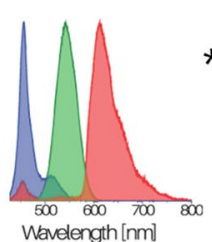

(c) QLED TV: LED backlit LCD TV with QD enhancement Film (QDEF-CF)

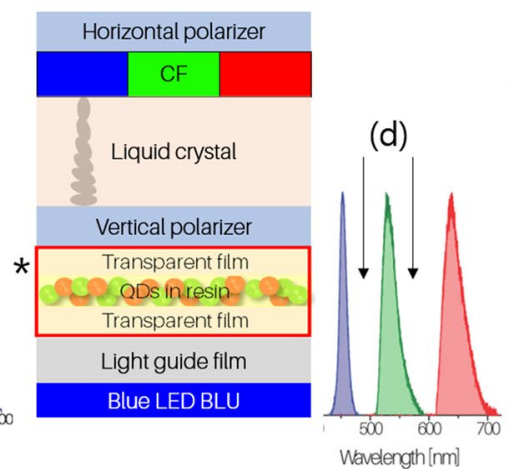

(e) New QLED concept with QD color filter (QD-CF)

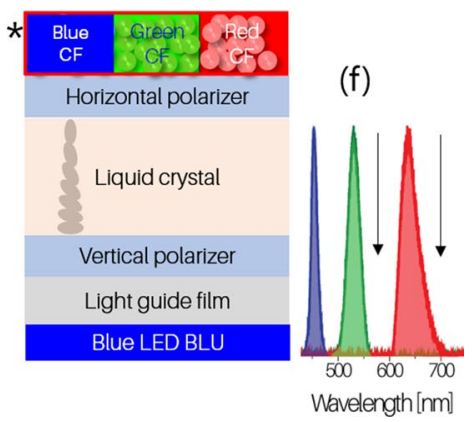

Figure 1. Different paradigms in LCD display. (a) White LED backlit LCD display also known as LED-TV. (b) Emission characteristics of the device in (a). (c) Blue LED backlit LCD display with QD enhancement film also known as QLED-TV. (d) Emission characteristics of the device in (c). (e) QD-color filter (QDCF) for QLED-TV. (f) Emission characteristics of the device in (e). The star symbol $\left({ }^{*}\right)$ indicates newly introduced parts of the device compared to preceding device. Each arrow $(\downarrow)$ in a spectrum indicates regions where the extent of overlap between PL emissions have been reduced compared to the previous spectrum.

future ${ }^{5,6}$. The recent trials of $8 \mathrm{~K}$ endoscopy equipment have demonstrated the usefulness of UHDTV formats in surgical procedures improving the level of detail accessible to the surgeon ${ }^{5}$.

The wider availability of UHDTV content will increase the demand for displays capable of handling their higher color gamut with a higher dynamic range. This requires new materials and fabrication strategies for displays. A solution being implemented in the market is the use of QDEF in combination with blue LED backlight unit (BLU) as a backlight in LCD displays ${ }^{6}$. The QDEF contains green and red QDs usually encapsulated in a poly(ethylene terephthalate) (PET) film. The green and red QDs absorb the light of the blue BLU and re-emit sharper green and red colored light, leading to a high-color gamut display ${ }^{7}$. Previous workers have employed Cadmium (Cd)-based, Indium (In)-based, and mixed halide perovskite QDs for QDEF layers ${ }^{8-11}$. The use of QDEF dramatically improves the color gamut of QD-LCD devices over conventional LCD devices. However, the proximity of quantum dots within the QDEFs are found to lead to cannibalistic absorption of light within QDEF films. Moreover, dipolar interaction between an excited green QDs with unexcited red QDs leads to fluorescent energy transfer (FRET) from green to red. Such energy transfer phenomena lead to red-biased emissions from the QDEF. This problem cannot be solved by reduction in the concentration of QDs to decrease interactions because it would lead to reduction in brightness ${ }^{9}$. It should be addressed by added components that help efficient light extraction from QDEF or by designing new device structures ${ }^{12}$.

We had recently demonstrated a new approach to enhancing the LCD display with perovskite QDs. Blue, green and red inorganic perovskite QDs were incorporated into the color filter element in the LCD to form a functional quantum dot incorporated color filter (QDCF)-to achieve lower overlap between RGB color elements in pixels ${ }^{8}$. Lead halide perovskites has high-intensity emissions with narrow full width at half-maximum $(\mathrm{FWHM})^{13}$. However, lead halide perovskite QDs are unsuited for mass market applications because of their instability, and presence of lead $(\mathrm{Pb})$ in them ${ }^{14}$. Indium-based quantum dots are better suited for commercial applications but their widespread application has been hampered due to the difficulty in synthetically controlling their complex crystallization behaviour and optical properties ${ }^{4,15}$.

Here we demonstrate the customization of PL emissions from environment friendly InPZnSe ${ }_{1-\mathrm{x}} \mathrm{S}_{\mathrm{x}} / \mathrm{ZnS}$ QDs and their use in QDCFs. The QDCF has green and red QDs specifically synthesized to have emissions with reduced FWHM, leading to less overlap and wider coverage of the Rec. 2020 color gamut. A summary of the concept of this work is presented in Fig. 1, where different types of devices and their polarized emissions are presented. A white LED backlit LCD display with a conventional color filter (CF) (here in after mentioned as LCD) is shown in Fig. 1a. There is a lot of crosstalk between the blue, green and red emissions in this device, as seen in the emission spectrum shown in Fig. 1b. The second device in Fig. 1c constitutes a blue LED backlit LCD display with a quantum dot enhancement film (QDEF), called QDEF-LCD from here on. This device features sharp emissions with decreased FWHM due to the introduction of QDEF in the device leading to reduced crosstalk between blue, green, and red emissions, as seen in the corresponding spectrum in Fig. 1d. The QDEF is made by QDs encapsulated in a polymer resin at high concentrations. The third device structure is our recently proposed paradigm for QLED devices ${ }^{8}$. It involves emission-tuned green and red $\mathrm{InPZnSe}_{1-\mathrm{x}} \mathrm{S}_{\mathrm{x}} / \mathrm{ZnS}$ QDs mixed with green and red color filters creating a quantum dot incorporated color filter (QDCF) element. Here QDCF substitutes the role of QDEF in the display, leading to an overall increase in emission intensity of the device while contributing to sharper green and red emission. This third type of device will be called QDCF LCD from here on. The device structure can be seen in Fig. 1e and the characteristic emission can be seen in Fig. 1f.

The interplay of components such as the backlight unit (BLU), the color filters, QDEF/QDCF, etc. in the display dictates the color and brightness delivered by the variants of LCD displays featured in Fig. 1. Color filters transmit a single color (red, green, or blue) from the emissions passing through them. A wide color gamut and high dynamic range results from the tandem action of both the emissive components and color filters. Overlaps 
(a)

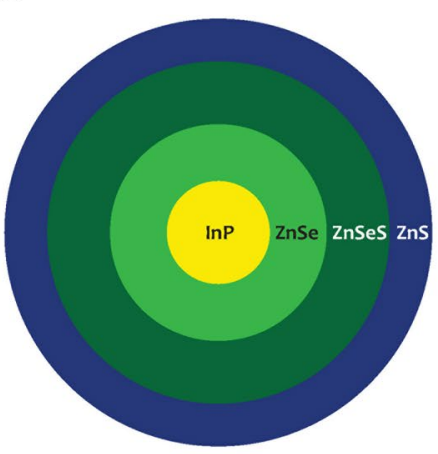

(c)

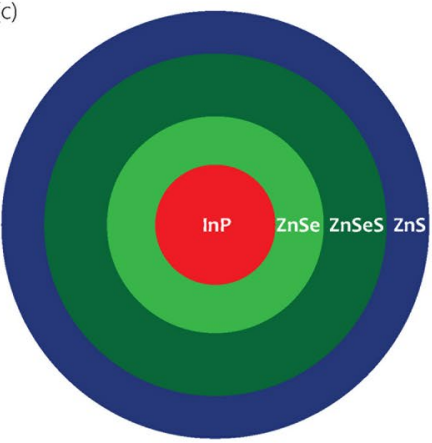

(b) Temporal sequence of added reactants with reaction temperatures

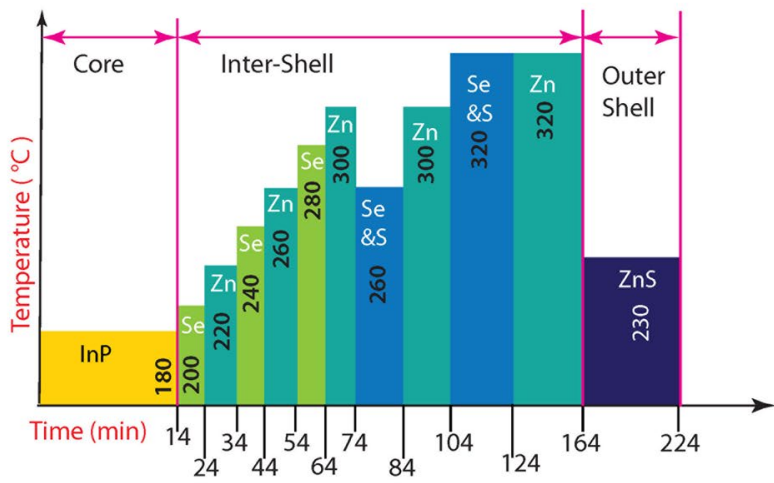

(d)

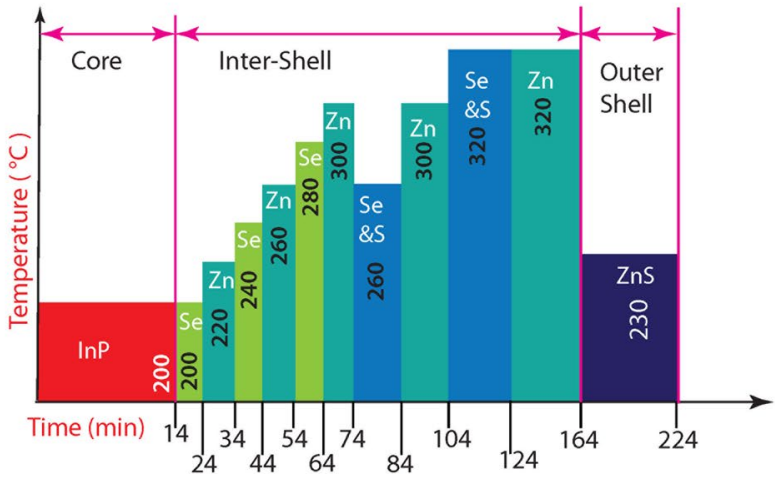

Figure 2. A summary of steps during the synthesis of InP/ZnSeS/ZnS QDs. (a) Illustration of composition gradient in the green InP/ZnSeS/ZnS (GQD) core/shell structure. (b) The temporal sequence of addition of different materials with corresponding temperatures during the synthesis of green QDs. (c) Illustration of composition gradient in the red InP/ZnSeS/ZnS (RQD) core/shell structure. (d) The temporal sequence of addition of different materials with corresponding temperatures during the synthesis of red QDs.

between PL emissions of blue, green, and red emissions give rise to crosstalk and reduces color gamut. In this work, the emissive properties of environmentally friendly InPZnSe ${ }_{1-x} S_{x} / Z n S$ QDs have been customized such that their incorporation in color filters leads to sharp, non-overlapping emissions and hence a wide color gamut.

\section{Results and discussion}

Environmentally friendly green quantum dot (GQD) and red quantum dot (RQD) with a generic core/shell structure of InPZnSe ${ }_{1-x} S_{x} / Z n S$ were synthesized for this work. The InP-based QDs (InP QDs) showed a high photoluminescence-quantum yield (PL-QY) and a narrow FWHM, as well as tunable absorption and emission properties to suit the peak transmitted wavelengths of a color filters used in LCDs. These QDs were synthesized by tuning the $\mathrm{In}^{3+}: \mathrm{P}^{3-}$ stoichiometric molar fraction in the core (of the QDs) to absorb the blue light from the LED backlight unit and emit only green, and red-light via energy-down-shift ${ }^{16}$. The QDs were synthesized by the successive ionic layer adsorption and reaction (SILAR) method. The summary of steps during the synthesis of green and red InPZnSe ${ }_{1-x} S_{x} / Z n S$ QDs is given in Fig. 2. The growth temperature for GQD and RQD cores was $180^{\circ} \mathrm{C}$ and $200^{\circ} \mathrm{C}$, respectively. The growth of the shell in both green and red QDs follows the same procedure involving the introduction of different ions at different temperatures, as seen in Fig. 2b,d. Such additions lead to a shell with a gradient concentration of Se moving from a Se-rich ZnSe on top of the core to an S-rich ZnS outer layer through an intermediate layer (interlayer) of gradually varying Se and S content. Hence, the inner layer and gradient layer compositions are expressed together as $\mathrm{ZnSe}_{1-\mathrm{x}} \mathrm{S}_{\mathrm{x}}$.

The difference in energy level between the core and the shell determines the quantum yield of fluorescence, while the extent of a lattice mismatch determines the FWHM of its emission spectrum. The gradient shell structure helps reduce the lattice mismatch within the core/shell structure ${ }^{17,18}$.

Both GQD and RQD were highly crystalline with low polydispersity, as seen in the HR-TEM images in Fig. 3a,b. The QDs had a cubical shape, unlike the Cd or InP-based core/shell QDs (i.e., spherical-like) commonly used in QDEF LCD ${ }^{16,19,20}$. The average sizes of GQD and RQD were $5.12 \mathrm{~nm}$ and $7.07 \mathrm{~nm}$, respectively. The GQD and RQD showed respective lattice constants of 5.8 and 5.9 $\AA$. The composition of the QDs determined by energy dispersive X-ray spectrometry (EDS) measurement are shown in the insets of Fig. 3a,b. InP QDs have a zinc blende crystalline structure (JCPDS No. 32-0452) ${ }^{21}$, which was confirmed by selected area electron diffraction (SAED) and X-ray diffraction (XRD) patterns (see Supplementary Figures S4a-d in the supporting information). 
(a)

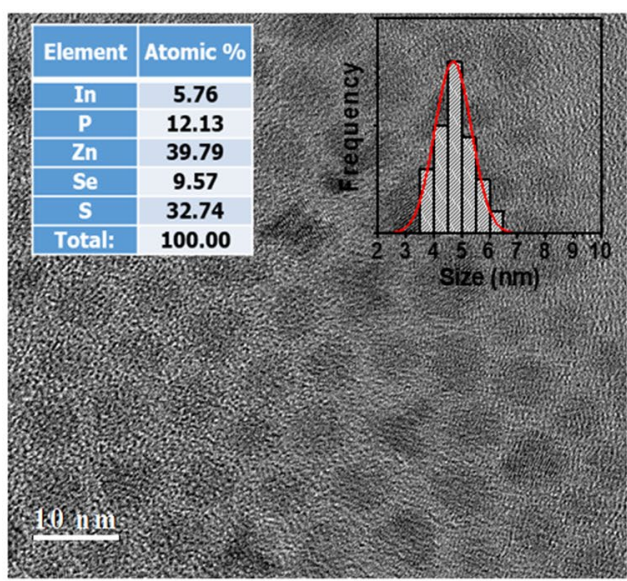

(c)

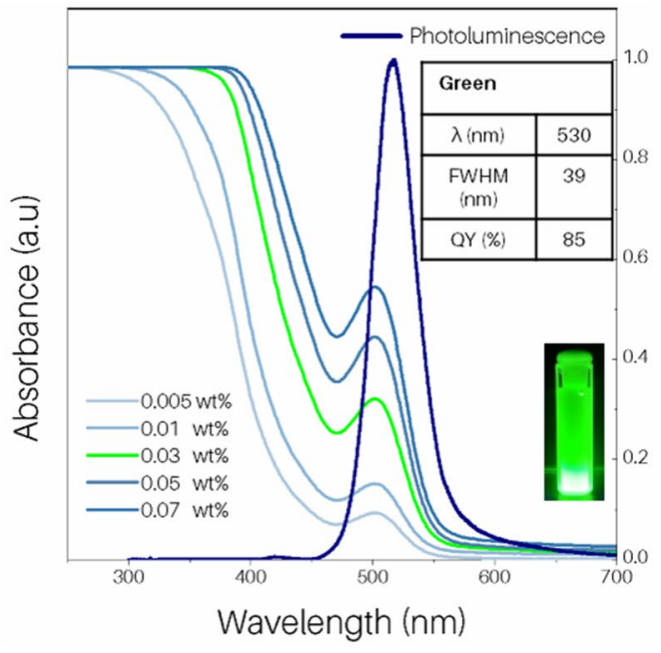

(b)

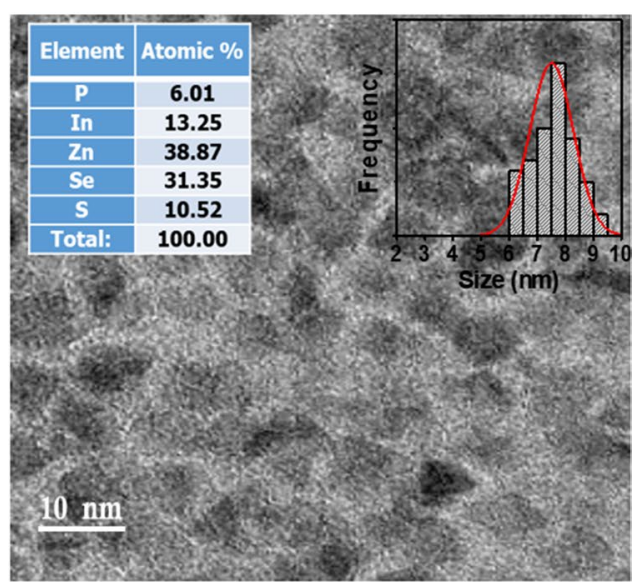

(d)

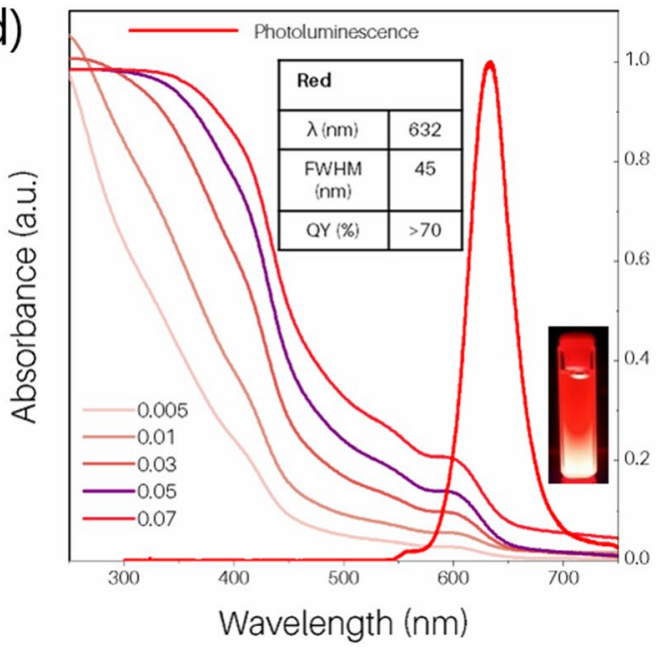

Figure 3. (a) TEM image of green QDs with size distribution. The inset summarizes the optical properties of the GQD. (b) TEM image of red QDs with size distribution. The inset summarizes the optical properties of the RQD. (c) Absorption spectrum corresponding to different concentrations of GQD nanoparticle dispersion, where the sharp curve on the right is the fluorescence spectrum. (d) Absorption spectrum corresponding to different concentrations of RQD nanoparticle dispersion. The inset summarizes the optical properties of the RQD, where the sharp curve on the right is the fluorescence and summarizes the optical properties of the RQD, and the sharp curve on the right is the fluorescence spectrum.

The distinct rings in the SAED spectrum originating from the 111,220, and 311 planes is quite consistent with the dominant XRD diffraction peaks for GQDs and RQDs. The larger size of RQDs leads to sharper XRD peaks compared to GQDs.

Note that gradient shell GQDs and RQDs described here have complex internal structures which could only be thoroughly characterized techniques such as X-ray photoelectron spectroscopy (XPS) or X-ray absorption fine structure study (XAFS) ${ }^{22,23}$. QDs are quite difficult to analyse with powder X-ray diffraction because of the small size of the nanoparticles effecting broadening of the spectrum. XPS has been used to study the extent and type of alloying in quantum dots by analysing the energies of the photoelectrons ${ }^{23}$. XAFS is more suitable for studying nanocrystals because it is independent of long-range ordering in crystals ${ }^{22}$.

The optical properties of GQD and RQD are summarized in Fig. 3c,d. The UV-vis absorption characteristics of the QDs were recorded at different concentrations ranging from 0.005 to $0.07 \mathrm{wt} \%$. Within this range of concentrations, the absorption peak intensities were found to increase with increasing concentration. The PL peak presented in both cases corresponds to a dispersion with a concentration of $0.07 \mathrm{wt} \%$. The emission spectrum of the GQD showed an emission maximum at $530 \mathrm{~nm}$ with an FWHM of $39 \mathrm{~nm}$ and quantum yield (QY) greater than $85 \%$. The RQDs showed an emission maximum at $632 \mathrm{~nm}$ with an FWHM of $45 \mathrm{~nm}$ and quantum yield (QY) greater than 70\%. The quantum yields were determined using time-resolved PL decay experiments. The QY for GQD and RQD was calculated from exciton lifetimes. The detailed calculation is summarized in Supplementary Figure S5 and Supplementary Table 1 in the supporting information.

The best color gamut performances are achieved in LCD devices when the blue, green, and red emissions fall well within the range of wavelengths transmitted by the blue, green and red color filters. Minimal spectral overlap between emissions after transmission through the color filters is crucial to the efficiency of these devices. In the case of the quantum dot-enhanced LCD devices discussed in this work, the blue emission originates from the 


\begin{tabular}{|c|c|c|c|c|c|c|}
\hline \multirow[b]{2}{*}{ Sample } & \multirow[b]{2}{*}{ Peak frequency } & \multirow[b]{2}{*}{ PL peak WL (nm) } & \multirow[b]{2}{*}{ FWHM (nm) } & \multirow[b]{2}{*}{ CIE coordinates $(x, y)$} & \multicolumn{2}{|c|}{ Color gamut } \\
\hline & & & & & NTSC[\%] & Rec. 2020[\%] \\
\hline \multirow{3}{*}{ Conventional LCD } & Blue & 456 & 20.6 & $(0.14,0.10)$ & \multirow{3}{*}{73.7} & \multirow{3}{*}{55.1} \\
\hline & Green & 541 & 49.1 & $(0.29,0.68)$ & & \\
\hline & Red & 612 & 58.7 & $(0.61,0.33)$ & & \\
\hline \multirow{3}{*}{ QDEF LCD } & Blue & 452 & 20.4 & $(0.15,0.03)$ & \multirow{3}{*}{95.2} & \multirow{3}{*}{71.2} \\
\hline & Green & 528 & 31.3 & $(0.24,0.70)$ & & \\
\hline & Red & 638 & 36.4 & $(0.64,0.30)$ & & \\
\hline \multirow{3}{*}{ QDCF LCD } & Blue & 452 & 20.4 & $(0.15,0.03)$ & \multirow{3}{*}{108.8} & \multirow{3}{*}{81.4} \\
\hline & Green & 530 & 26.1 & $(0.18,0.74)$ & & \\
\hline & Red & 638 & 36.7 & $(0.65,0.30)$ & & \\
\hline
\end{tabular}

Table 1. Optical characteristics of LCD, QDEF-LCD and QDCF-LCD.
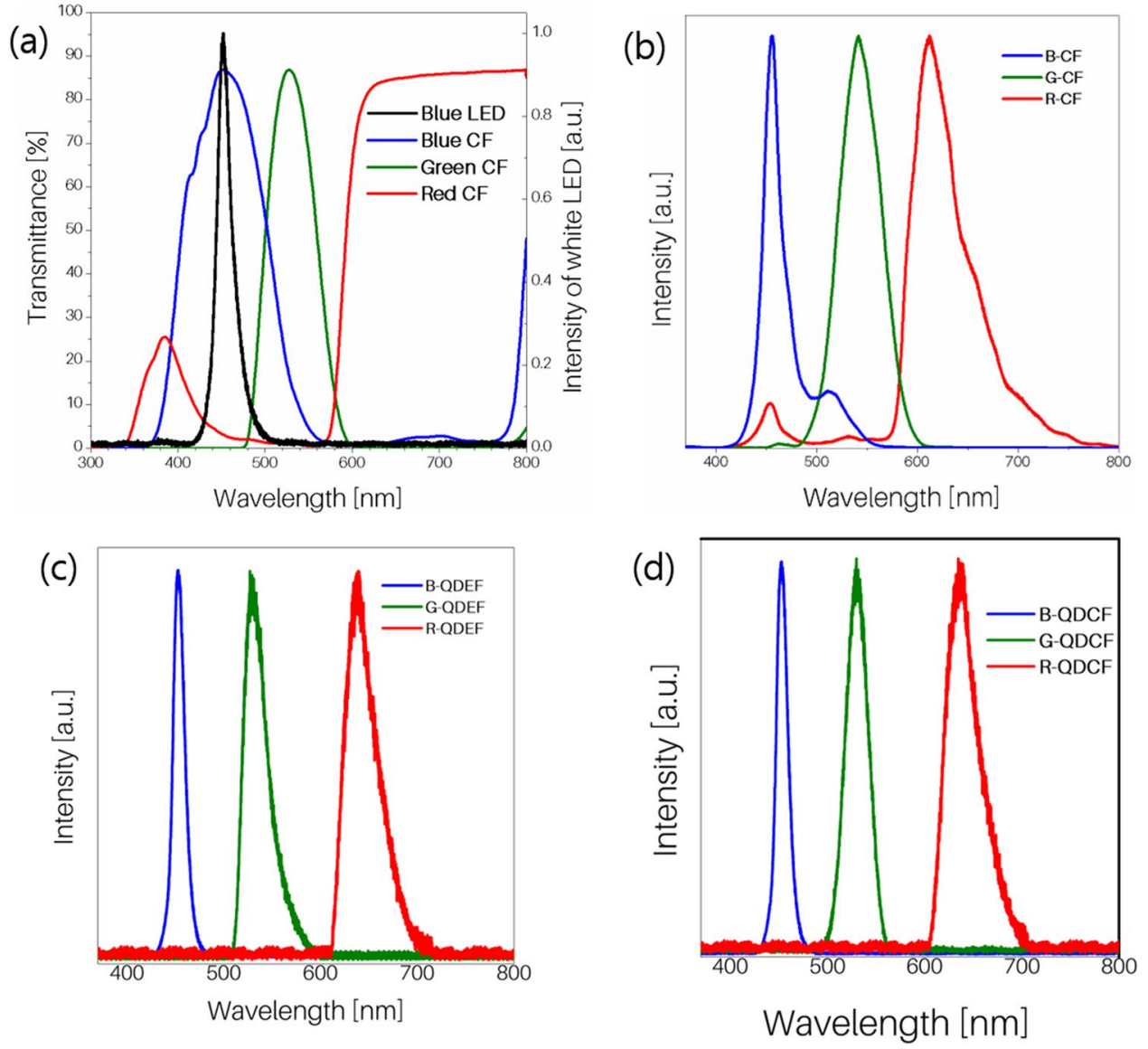

Figure 4. Optical characteristics of various components constituting the fabricated displays. (a) Emission properties of the BLU and color filter (sharp black curve) to the percentage transmittance of blue, green and red color filters. RGB emission of (b) white LED Backlit LCD (c) BLU backlit LCD device with QDEF (d) BLU backlit LCD device with quantum dot integrated color filters (QDCF).

blue LED backlight unit (BLU). The emissive characteristics of the BLU, giving rise to an emission maximum of $452 \mathrm{~nm}$ as seen in Fig. 4a. This was measured by arranging a blue LED from the BLU in the PL spectrometer. The emission spectrum of the blue color filter was measured by coating it on a quartz slide and using it as a sample to measure fluorescence. The same procedure using the quartz substrate was followed in the case of green and red color filters. The range of wavelengths transmitted by the blue $\left(371-563 \mathrm{~nm}, \lambda_{\max }=451 \mathrm{~nm}\right)$, green $(478-595 \mathrm{~nm}$, $\left.\lambda_{\max }=527 \mathrm{~nm}\right)$ and red $\left(>570 \mathrm{~nm}, \lambda_{\max }=631 \mathrm{~nm}\right)$ color filters used in LCD devices are represented by the blue, green and red curves in Fig. $4 \mathrm{a}$, respectively. 


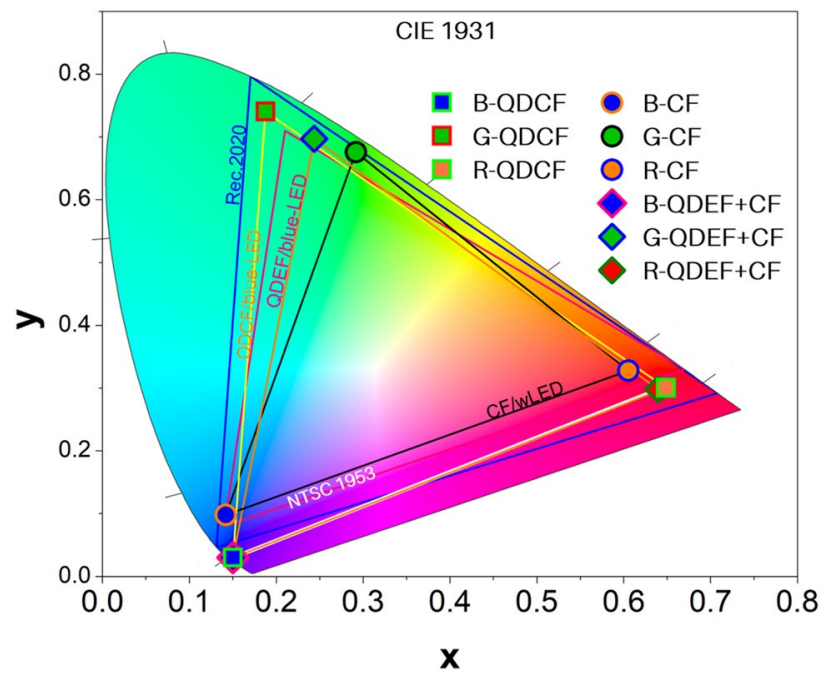

Figure 5. Comparison of the RGB color triangles formed by the CIE (CIE 1931) coordinates of blue, green, and red polarized PL emissions of conventional LCD, QDEF-LCD and QDCF-LCD. The color gamut defined by the NTSC 1953 standard is represented as a pink triangle, while the color gamut of Rec.2020 standard is represented by the blue triangle.

As mentioned earlier, three different LCD device structures were fabricated for this study, namely LCD, QDEF-LCD and QDCF-LCD. The first one is a white LED BLU-lighted LCD display with a conventional device structure, as seen in Fig. 1b; the second one, QDEF-LCD, is a blue BLU-lit QD-enhanced LCD with QDEF, as seen in Fig. 1c; and the third one, QDCF-LCD, is a blue backlit QD-enhanced LCD with a QDCF, as shown in Fig. 1d. The conventional LCD device gave polarized blue emissions at $456 \mathrm{~nm}$ with an FWHM of $20.6 \mathrm{~nm}$, the green emission had a peak wavelength of $541 \mathrm{~nm}$ with an FWHM of $49.1 \mathrm{~nm}$. The red color polarized fluorescence from the above device was at $612 \mathrm{~nm}$ with an FWHM of $58.7 \mathrm{~nm}$. The PL emission of the conventional LCD device given in Fig. 4b. shows considerable polarized PL spectrum crosstalk (overlap) between red, green and blue emission spectra. The spectral characteristics are summarized in Table 1.

The polarized blue, green and red emissions from the QDEF-LCD can be seen in Fig. 4c. The polarized blue emission from QDEF-LCD device peaked at $452 \mathrm{~nm}$ with an FWHM of 20.4. The polarized green emission peaked at $528 \mathrm{~nm}$ with an FWHM of $31.3 \mathrm{~nm}$. The polarized red emission peaked at $638 \mathrm{~nm}$ with an FWHM of $36.4 \mathrm{~nm}$. In Fig. 4c, the polarized PL emissions from the QDEF-LCD are almost free of crosstalk because there is little or no overlap between the emissions. This leads to a considerable improvement in the color gamut of the LCD.

The QDCF-LCDs were fabricated by integrating GQD and RQD into green and red color filters, respectively. The direct integration of quantum dots into the color filters means that QDCF-LCD devices does not need a QDEF (compare devices in Fig. 1c,e). The emission wavelength $\left(\lambda_{\max }\right)$ and FWHM governs the color gamut of a display ${ }^{24}$. Incorporation of QDs into devices as QDCFs increases the color gamut by improving the fwhm of GQDs. The polarized blue, green and red PL emissions from the QDCF-LCD can be seen in Fig. 4d. The blue emissions peaked at $452 \mathrm{~nm}$ with an FWHM of $20.4 \mathrm{~nm}$. The green emissions peaked at $530 \mathrm{~nm}$ with an FWHM of $26.1 \mathrm{~nm}$. The red emissions peaked at $638 \mathrm{~nm}$ with an FWHM of $36.7 \mathrm{~nm}$. In the tabulated FWHM data in Table 1, green and red emissions in the QDCF-LCD shows a considerable reduction compared to the conventional LCD device. The blue emission of the QDEF-LCD and the QDCF-LCD was slightly blue-shifted compared to the LCD display because of the difference in backlight units. The blue emissions from all three devices show similar narrow FWHM of around $20 \mathrm{~nm}$. The FWHM of the green emission decreases in the QDCF-LCD compared to the QDEF-LCD, leading to the possibility of a wider color gamut in the former. The emission maximum of the GQD in solution is $530 \mathrm{~nm}$. When the GQD is integrated into the green color filter in the QDCF-LCD the range of wavelengths available for the QD is controlled by the wavelength filtering action of the color filter compound $\left(478-595 \mathrm{~nm}, \lambda_{\max }=527 \mathrm{~nm}\right)$. Hence, the combination emits at a peak wavelength of $530 \mathrm{~nm}$. The red shift in green emission peaks of the QDCF-LCD compared to the QDEF-LCD may be due to the restriction on absorption wavelengths placed by the color filter. The absorption spectrum of the GQD in solution is compared with the transmitted wavelengths of a green color filter and the emission of a QD integrated green color filter in Supporting Figure S6(a). Similarly, the red color filter restricts the incoming wavelengths available for the red QDs in the red QDCF to wavelengths greater than $570 \mathrm{~nm}$. The emissions peak of RQD incorporated into QDCF is at $638 \mathrm{~nm}$. The red emission maximum in the QDCF-LCD is the same as the polarized red emission from the QDEF-LCD. The absorption spectrum of the RQD in solution is compared with the transmitted wavelengths of the red color filter and the emission of the QD integrated red color filter in Supporting Figure S6(b).

The color gamut performance of the three different displays was studied in order to assess their potential as ultra-high-resolution LCD displays. The performances of LCD, QDEF-LCD and QDCF-LCD were compared by estimating their coordinates in the CIE 1931 color space using two different standards, namely NTSC 1953 
and Rec.2020. Among these, the specification for UHD displays falls in the Rec.2020 color space. The CIE coordinates of the three types of LCDs were calculated from their PL spectra in Fig. 4b,d and a MATLAB-based CIE coordinate calculator. The calculated CIE coordinates for all devices are summarized in Table 1 . The two standard color spaces describe triangular graphs in the CIE color space, as seen in Fig. 5. The triangle of a standard in the CIE space encompasses all colors that could be expressed by a display that complies with that standard.

The RGB color gamut of the conventional LCD is $73.7 \%$ of the NTSC standard and $55.1 \%$ of the Rec. 2020 standard. The coordinates calculated from the emission of a conventional LCD are shown as closed circles. The triangle formed by the coordinates of the LCD in the blue, green and red regions of the color space fills only $73.7 \%$ of the NTSC standard color space and further falls short by covering only $55.1 \%$ of the Rec. 2020 color space. This means that this display is more suited for standard resolution displays than UHD displays. The CIE co-ordinates of the QDEF-LCD are represented as diamond $(\diamond)$ symbols in Fig. 5. The QDEF-LCDs cover respectively cover $95.2 \%$ of the NTSC colorspace and $71.2 \%$ of the REC 2020 color space. The standard wavelength corresponding to blue, green, and red colors in REC.2020 standard are $467 \mathrm{~nm}, 532 \mathrm{~nm}$ and $630 \mathrm{~nm}$ respectively. The emissions of the QDEF-LCDs corresponding to blue, green, and red are $452 \mathrm{~nm}, 528 \mathrm{~nm}$ and $638 \mathrm{~nm}$ respectively. Previous studies comparing the performance of red InP QDs have established that they show high REC.2020 color gamut performance when the red emission was at $652.1 \mathrm{~nm}$; when the red wavelength is decreased to match the standard $630 \mathrm{~nm}$ the gamut performance fell well below $90 \%{ }^{10}$. Hence improving the performance of red color is important in improving the REC.2020 performance of the device. Such an improvement would result from closely understanding the interaction between the QD emissions and the color filter and optimizing their interaction. However, when QDs were incorporated in the device in the form of QDCF an improved performance of $108.8 \%$ in the NTSC color space and $81.4 \%$ of REC 2020 colorspace was obtained. This broad coverage offered by QDCF in both NTSC and REC 2020 colorspaces enable QDCF LCDs to express greater color and dynamic range compared to LCDs and QDEF LCDs. We have earlier reported an RGB color gamut of $100.4 \%$ for NTSC standard and 134.2\% for REC 2020 standard for QDCF LCD devices based on perovskites ${ }^{8}$. In another work involving perovskite QDs Lin and co-workers reported 158.93\% of NTSC and 118.60\% of Rec. 2020 respectively ${ }^{25}$. However, there are no reports of QDCF type devices with InP based quantum dots showing high performances described here. The dramatic enhancement in the green emission in QDCFs compared to QDEF results from the synergistic action of the green color filter and the GQD emissions. The excitation wavelengths available from the GQDs in the QDCF are expressed by the overlap of the GQD absorbance spectrum and the transmission spectrum of green color filter in Figure s6a. This overlap results in a sharp green emission $(530 \mathrm{~nm})$ close to the Rec.2020 green wavelength standard $532 \mathrm{~nm}$. The red emission from the QDCF device $(638 \mathrm{~nm})$ deviates to a greater extent from the Rec.2020 red wavelength standard $630 \mathrm{~nm}$. The red color emission also shows broader FWHM due broader overlap between the red color filter and absorption of the RQD seen in Figure S6b. Developing a better understanding of the interaction between the QDs and the QDCFs will lead to even better devices of this genre in the future.

\section{Conclusions}

Synthesis of highly bright cadmium-free red and green InPZnSe ${ }_{1-x} S_{x} / Z n S$ QDs and their application in UHD LCDs have been demonstrated in this work. The synthetic tuning of QDs with gradient interlayer $\left(\mathrm{ZnSe}_{1-\mathrm{X}} \mathrm{S}_{\mathrm{X}}\right)$ leads to highly bright QDs. The green QD has a quantum yield of 85\% (FWHM $26.1 \mathrm{~nm}$ ) and the red QD has quantum yield of 70\% (FWHM 36.7) respectively. The use of QDs in QDCFs ushers in the third paradigm shift in LCD technology. It overcomes the limitations of QDEFs like the loss of optical power due to encapsulation driven interaction of QDs. The apparent optical output from QDCFs also depend on the color filter materials because the color filter governs the wavelengths accessible during QD excitation by blue backlight. The color gamut performance of the QDCF LCDs improves due to the reduced FWHM of green QDs. Crucially high color gamut is perceived as brightness by the human eye due to Helmholtz-Kohlrausch Effect ${ }^{26}$. We believe that further investigation to understand the interaction between the red QD and the corresponding color filter could lead to improved red emissions and push the QDCF devices towards full REC 2020 compliance.

\section{Data availability}

All data generated or analysed during this study are included in this published article (and its Supplementary Information files). Further data associated with publication is available from the authors upon reasonable request.

Received: 26 February 2020; Accepted: 24 August 2020

Published online: 25 September 2020

\section{References}

1. Konstantatos, G. \& Sargent, E. H. Colloidal Quantum Dot Optoelectronics and Photovoltaics (Cambridge University Press, Cambridge, 2013).

2. Basché, T. et al. Energy and charge transfer in nanoscale hybrid materials. Macromol. Rapid Commun. 36, 1026-1046 (2015).

3. Prabhakaran, P., Kim, W. J., Lee, K.-S. \& Prasad, P. N. Quantum dots (QDs) for photonic applications. Opt. Mater. Expess 2, 578-593 (2012).

4. Tamang, S., Lincheneau, C., Hermans, Y., Jeong, S. \& Reiss, P. Chemistry of InP nanocrystal syntheses. Chem. Mater. 28, 2491-2506 (2016).

5. Yamashita, H., Aoki, H., Tanioka, K., Mori, T. \& Chiba, T. Ultra-high definition (8K UHD) endoscope: our first clinical success. SpringerPlus 5, 1445 (2016).

6. Luo, Z., Xu, D. \& Wu, S.-T. Emerging quantum-dots-enhanced LCDs. J. Disp. Technol. 10, 526-539 (2014).

7. Kim, H.-J., Shin, M.-H., Lee, J.-Y., Kim, J.-H. \& Kim, Y.-J. Realization of $95 \%$ of the Rec. 2020 color gamut in a highly efficient LCD using a patterned quantum dot film. Opt. Express 25, 10724-10734 (2017). 
8. Ko, Y.-H., Jalalah, M., Lee, S.-J. \& Park, J.-G. Super ultra-high resolution liquid-crystal-display using perovskite quantum-dot functional color-filters. Sci. Rep. 8, 12881 (2018).

9. Chen, C.-J., Lin, C.-C., Lien, J.-Y., Wang, S.-L. \& Chiang, R.-K. Preparation of quantum dot/polymer light conversion films with alleviated Förster resonance energy transfer redshift. J. Mater. Chem. C 3, 196-203 (2015).

10. Lee, E. et al. SID Symposium Digest of Technical Papers. 549-551 (Wiley Online Library).

11. Ko, Y.-H. \& Park, J.-G. Novel quantum dot enhancement film with a super-wide color gamut for LCD displays. J. Korean Phys. Soc. 72, 45-51 (2018).

12. Yang, X. et al. Light extraction efficiency enhancement of colloidal quantum dot light-emitting diodes using large-scale nanopillar arrays. Adv. Funct. Mater. 24, 5977-5984 (2014).

13. Swarnkar, A. et al. Colloidal CsPbBr 3 perovskite nanocrystals: luminescence beyond traditional quantum dots. Angew. Chem. 127, 15644-15648 (2015).

14. Berhe, T. A. et al. Organometal halide perovskite solar cells: degradation and stability. Energy Environ. Sci. 9, 323-356 (2016).

15. Allen, P. M., Walker, B. J. \& Bawendi, M. G. Mechanistic insights into the formation of InP quantum dots. Angew. Chem. 49, 760-762 (2010).

16. Baek, S.-W. et al. Low-cost and flexible ultra-thin silicon solar cell implemented with energy-down-shift via Cd $0.5 \mathrm{Zn} 0.5 \mathrm{~S} / \mathrm{ZnS}$ core/shell quantum dots. J. Mater. Chem. A 3, 481-487 (2015).

17. Lim, J. et al. InP@ZnSeS, Core@Composition gradient shell quantum dots with enhanced stability. Chem. Mater. 23, 4459-4463 (2011).

18. Bae, W. K., Char, K., Hur, H. \& Lee, S. Single-step synthesis of quantum dots with chemical composition gradients. Chem. Mater. 20, 531-539 (2008).

19. Jalalah, M., Ko, Y.-H., Harraz, F. A., Al-Assiri, M. \& Park, J.-G. Enhanced efficiency and current density of solar cells via energydown-shift having energy-tuning-effect of highly UV-light-harvesting $\mathrm{Mn}^{2+}$-doped quantum dots. Nano Energy 33, 257-265 (2017).

20. Kim, S. et al. Highly luminescent InP/GaP/ZnS nanocrystals and their application to white light-emitting diodes. J. Am. Chem. Soc. 134, 3804-3809 (2012).

21. Liu, Z., Bai, Y., Cui, D. \& Wang, Q. Preliminary insight into the formation process of InP and GaP nanocrystals. Solid State Sci. 5, 1037-1040 (2003).

22. Rao, M. J., Shibata, T., Chattopadhyay, S. \& Nag, A. Origin of photoluminescence and XAFS study of $(\mathrm{ZnS})_{1-\mathrm{x}}(\operatorname{AgInS} 2)_{\mathrm{x}}$ nanocrystals. J. Phys. Chem. Lett. 5, 167-173 (2014).

23. Sarma, D., Santra, P. K., Mukherjee, S. \& Nag, A. X-ray photoelectron spectroscopy: a unique tool to determine the internal heterostructure of nanoparticles. Chem. Mater. 25, 1222-1232 (2013).

24. Coe-Sullivan, S., Liu, W., Allen, P. \& Steckel, J. S. Quantum dots for LED downconversion in display applications. ECS J. Solid State Sc. 2, R3026-R3030 (2013).

25. Lin, S. et al. Multi-primary-color quantum-dot down-converting films for display applications. Opt. Express 27, 28480-28493 (2019).

26. Chen, H., He, J. \& Wu, S.-T. Recent advances on quantum-dot-enhanced liquid-crystal displays. IEEE J. Sel. Top. Quantum Electron. 23, 1-11 (2017).

\section{Acknowledgements}

This work was supported by the 2019 Industry-Academia Cooperation Program of SAMSUNG DISPLAY, the Mid-Career Researcher Program of the National Research Foundation (NRF) funded by the MEST (No. 2016R1A2B4008473) and the Basic Science Research Program (2017R1C1B5077130) of the National Research Foundation (NRF) of Korea.

\section{Author contributions}

Y.-H.K., and P.P. conceived and carried out majority of the experiments. S.C. and G.-J.K. assisted the experiments and characterizations. K.-S.L supervised the materials synthesis. C.L. supervised the fabrication of devices and their characterization. P.P. and Y.-H.K. wrote the paper wrote the paper and all co-authors edited it. All authors have given approval to the final version of the manuscript.

\section{Competing interests}

The authors declare no competing interests.

\section{Additional information}

Supplementary information is available for this paper at https://doi.org/10.1038/s41598-020-72468-8.

Correspondence and requests for materials should be addressed to C.L. or K.-S.L.

Reprints and permissions information is available at www.nature.com/reprints.

Publisher's note Springer Nature remains neutral with regard to jurisdictional claims in published maps and institutional affiliations.

(c) (i) Open Access This article is licensed under a Creative Commons Attribution 4.0 International License, which permits use, sharing, adaptation, distribution and reproduction in any medium or format, as long as you give appropriate credit to the original author(s) and the source, provide a link to the Creative Commons licence, and indicate if changes were made. The images or other third party material in this article are included in the article's Creative Commons licence, unless indicated otherwise in a credit line to the material. If material is not included in the article's Creative Commons licence and your intended use is not permitted by statutory regulation or exceeds the permitted use, you will need to obtain permission directly from the copyright holder. To view a copy of this licence, visit http://creativecommons.org/licenses/by/4.0/.

(C) The Author(s) 2020 